\title{
Editorial
}

\section{Cáncer de ovario, ¿podemos clasificar las masas anexiales complejas por ecografía?}

\author{
Dr. Nelson Burgos Siegmund
}

Ecografísta Ginecológico. Departamento de Obstetricia y Ginecología sede Norte. Unidad de Ecografía Ginecológica. Hospital Clínico de la Universidad de Chile. Servicio de Ecografía Ginecológica Clínica Alemana de Santiago. Unidad de Imagenología de la Mujer, Clínica Indisa.

El cáncer de ovario epitelial es la neoplasia ginecológica más letal a nivel mundial y también en Chile ${ }^{1}$. Mundialmente se diagnostican 238.800 nuevos casos anuales (2012), con 175.982 muertes el año en 2017, en el lugar 14 de las muertes por cáncer, con una tasa de mortalidad de 4/100.0002. La tasa de sobrevida a 5 años en EUA es de $46,5 \%$ al periodo 2007-20132. En Chile la tasa cruda de mortalidad por 100.000 ha aumentado de 3,4 en 1997 a 4,8 en 2015, con una incidencia de 6,7/100.000 entre 2003 a $2007^{3}$.

En 2013 se incorporan 11 nuevas patologías al plan AUGE (Acceso Universal de Garantías Explícitas), por la Ley 19.996 Decreto $\mathrm{N}^{\circ} 4$ de la República de Chile, donde se incorpora el cáncer de ovario epitelial en sus distintas etapas ${ }^{1}$. Dentro de los indicadores de derivación, desde la atención primaria (Médico de consultorio o Isapre), al especialista gineco-obstetra capacitado o gineco-oncólogo esta el diagnóstico ecografía que se denomina "Masa anexial compleja o sólido", para la mujer pre o postmenopáusica mediante "ecografía abdominal y pélvica"4.

Desde la aparición de la ecografía se han buscado los hallazgos que pueden caracterizar una lesión anexial, tratando de diferenciar lo benigno de lo maligno. Es así como el año 2000 se publica un consenso de opinión del grupo Internacional de Análisis de los Tumores Ováricos (IOTA), que describe los términos, definiciones y mediciones de las características ecográficas de los tumores anexiales, esta publicación tiene como objetivo dar las bases para la evaluación y la investigación de las lesiones anexiales 5 . Desde esa fecha la gran mayoría de las publicaciones utilizan esta descripción $6,7,8$.
El grupo IOTA ha trabajado desde ese año 2000 en poder diferenciar las lesiones anexiales benignas de las malignas, mediante la propuesta de diferentes modelos predictivos como: regresión logística (LR1 y LR2), reglas simples, descriptores instantáneos ${ }^{9}$, ADNEX ${ }^{10}$, simples rules risk ${ }^{11}$ e incluso seguimiento de lesiones de aspecto benignos y sus posibles complicaciones ${ }^{12}$.

El año 2005 en Chile, Amor y otros ${ }^{13}$, publica la buena sensibilidad y especificidad que tiene la ecografía en manos de operadores entrenados, para el diagnóstico presuntivo de anaplasia en las "masas anexiales complejas". El año 2007 Amor y Vaccaro'14 proponen una clasificación e informe de las imágenes anexiales por ultrasonografía transvaginal utilizando la sigla GIRADS (Gynaecological Imaging Reporting And Data System), Separando los hallazgos en 5 grupos, estableciendo para cada grupo las imágenes que se deben incorporar y estableciendo el riesgo de malignidad para c/u. En los grupos 4 y 5 están las pacientes con riesgo de malignidad. Proponen además que se incorpore esta categorización en los informes. El mismo grupo en dos publicaciones internacionales el año 2009 con datos nacionales de 187 masas anexilaes 6 y el 2011 con estudio multicéntrico internacional de 432 masas anexiales ${ }^{7}$, En ambos trabajos se utiliza como caracterización morfológica lo propuesto por IOTA. Recomiendan para cada GIRADS una conducta, que puede ser: seguimiento, manejo por gine-general, gine-oncólogo, o estudios complementarios ${ }^{6,7}$. Estás dos publicaciones permitieron que GIRADS sea utilizada en otros países. El Colegio Americano de Radiólogos ha publicado una nueva clasificación de las masas 
anexiales, separándolas en 5 grupos que llaman ORADS, el grupo analizó a GIRADS, pero definitivamente utilizan los datos de IOTA, por la mayor casuística e investigación tras IOTA ${ }^{8}$.

Luego de 10 años de GIRADS, para clasificar las lesiones anexiales, la propuesta del sistema ha sido utilizado por varios centros en el mundo. En este número de la Revista se presenta: GI-RADS: Método de Clasificación de Masas Anexiales, Revisión Sistemática y Metanálisis. 10 años de Experiencia.

En la revisión se identificaron 15 citas, incluyendo 13 estudios con 4473 masas, de las cuales 878 fueron malignas, prevalencia media de malignidad de $23 \%$ y agrupada de $19,6 \%$. El promedio de edad entre 37,5 y 47,2 años. El sistema presento una sensibilidad de $96,8 \%$, especificidad de $91,2 \%$, probabilidad positiva de 11.0 y negativa de 0,035 .

De los 13 estudios incluidos en la revisión 8 de ellos fueron prospectivos y 5 retrospectivos. En el dominio "prueba índice", todos los estudios describieron adecuadamente el método de texto índice, como se realizó y como se interpretó la clasificación de GIRADS.

La distribución de las distintas categorías de GIRADS resultantes fueron: GI-RADS 1-2 no hay cánceres, GI-RADS 3 un 1,9\%, GI-RADS 4 un 53.7\% y en GI-RADS 5 un $90 \%$ son cánceres. El tener un GlRADS 4-5 aumenta la probabilidad de tener un cáncer de ovario de $22,6 \%$ como riesgo basal a $76,0 \%$, mientras que un GI-RADS 1-3 disminuye de $22,6 \%$ a $1,0 \%$.

Se realizaron en Europa, Asia, África, América del Norte y América del Sur, aplicado en distintas poblaciones.

Un objetivo principal de GI-RADS es informar y facilitar la comunicación con el tratante, esto fue analizado en dos estudios, donde se considero de utilidad para ellos.

Los autores resaltan que este método no es una forma para interpretar el hallazgo ecográfico, sino que un sistema estructurado de informe para mejorar la comunicación entre el ecografísta y los tratantes, proponiendo un diagnóstico etiológico y/o un riesgo de malignidad. Se basa en las características subjetivas por un examinador experto. El método Gi-RADS es capaz de clasificar todas las lesiones anexiales, y se pueden obtener resultados comparables entre los operadores.

Esta revisión sistémica y metanálisis de GI-RADS confirma que tenemos un método que es efectivo en la clasificación de las lesiones anexiales, pudiendo diferenciar lo benigno de lo maligno y darle un nombre a la lesión que estamos evaluando.

El grupo IOTA, ha progresado en la evaluación de las masas anexiales, dejando cada vez menos masas sin clasificar, teniendo muy buen resultado en catalogar una lesión como benigna o maligna y determinar si estamos antes un borderline, un cáncer en estadio precoz o avanzado ${ }^{10,11}$.

GI-RADS, IOTA y los ecografístas experimentados, han demostrado tener las herramientas ecográficas para poder clasificar las lesiones anexiales, y no utilizar más en los informes ecográficos el término "Quiste, masa o lesión anexial compleja", donde pueden entrar muchos diagnósticos benignos.

Es nuestra responsabilidad como ecografístas especializados, junto a las Sociedades Científicas, Las Universidades y el Estado mediante el MINSAL ${ }^{1}$, educar a los ginecólogos en las características ecográficas para darle un nombre a la lesión anexiales.

\section{REFERENCIAS}

1. Cuello M. GES en cáncer de ovario epitelial: un avance sanitario necesario, pero no exento de riesgos y dificultades futuras. Editorial. Rev Chil Obstet Ginecol 2013; 78(3): 161-166.

2. Global Burden of Disease Collaborative Network. Global Burden of Disease Study 2017 (GBD 2017) Results. Seattle, United States: Institute for Health Metrics and Evaluation (IHME), 2018. http://ghdx.healthdata.org/gbd-results-tool

3. MINSAL. Plan Nacional de Cáncer 2018-2028. 2019: 1-185.

4. MINSAL. Guía Clínica AUGE. Cáncer de Ovario Epitelial. 2013: 1-53.

5. Timmerman D., Valentin L., Bourne T.H., Collins W.P., Verrelst $H$. and Vergote I. Terms, definitions and measurements to describe the sonographic features of adnexal tumors. A consensus opinion the International Ovarian Tumor Analysis (IOTA) group. Ultrasound Obstet Gynecol 2000; 16: 500505.

6. Amor F., Vaccaro H., Alcazar J.L., León M., Craig J. M. and Martinez J. Gynecologic Imaging Reporting and Data System. A New Proposal for Classifying Adnexal Masses on the Basis of Sonographic Findings. J Ultrasound Med 2009; 28:285-291. 
7. Amor F., Alcazar J.L., Vaccaro H., León M. and Iturra A. GIRADS reporting system for ultrasound evaluation of adnexal masses in clinical practice: a prospective multicenter study. Ultrasound Obstet Gynecol 2011; 38: 450-455.

8. Andreotti R., Timmerman D., Strachowski L., Froyman W., Benacerraf B., Bennet G., Bourne T., Brown D., Coleman B., Frates M., Goldstein S., Hamper U., Horrow M., Hernanz-Schulman M., Reinhold C., Rose S., Whitcomb B., Wolfman W. and Glanc P. O-RADS US Risk Stratification and Management System: A Consensus Guideline from the ACR Ovarian-Adnexal Reporting and Data System Committee. Radiology 2019; 00: 1-18.

9. Kaijser J., Bourne T., Valentin L., Sayasneh A., Van Holsbeke C., Vergote I., Testa A. C., Franchi D., Van Calster B. and Timmerman D. Improving strategies for diagnosing ovarian cancer: a summary of the International Ovarian Tumor Analysis (IOTA) studies. Ultrasound Obstet Gynecol 2013; 41: 9-20.

10. Van Calster B., Van Hoorde K., Valentin L., Testa A. C., Fischerova D., Van Holsbeke C., Savelli L., Franchi D., Epstein E., Kaijser J., Van Belle V., Czekierdowski A., Guerriero S., Fruscio R., Lanzani Ch., Scala F., Bourne T., Timmerman D. IOTA group. Evaluating the risk of ovarian cancer before surgery using the ADNEX model to differentiate between benign, borderline, early and advanced stange invasive, and secondary metastatic tumours: prospective multicentre diagnostic study. BMJ 2014;349:g5920 doi: 10.1 136bmj.g5820.

11. Timmerman D., Van Calster B., Testa A., Savelli L., Fischerova D., Froyman W., Wynants L., Van Holsbeke C., Epstein E., Franchi D., Kaijer J., Czekierdowski A., Guerriero S., Friscio R., Leone F., Rossi A., Landolfo Ch., Vergote I., Bourner T. and Valentin L. Predicting the risk of malignancy in adnexal masses based on the Simple Rules from the International Ovarian Tumor Analysis group. American Journal of Obstetrics Gynecology 2016: 424-437. Ajog.org.

12. Froyman W., Landolfo Ah., De Cock B., Wynants L., Sladkevicius P., Testa A., Van Hosbeke C., Domali E., Fruscio R., Epstein E., Dos Santos M., Franchi D., Kurla M., Chiappa V., Alcazar J.L., Leone F., Buonomo F., Hochberg L., Coccia M., Guerriero S., Deo N., Jokubkiene L., Kaijser J.,
Coosemens A., Vergote I., Verbakel J., Bourne T., Van Calster B., Valentin L. and Timmerman D. Risk of complications in patients with conservatively managed ovarian tumours (IOTA5): a 2-year interim analysis of a multicentre, prospective cohort study. The Lancet Oncology 2019; 20 (3): 448-458.

13. Amor F., Vaccaro H., Martinez J., Iturra A. and Zúñiga I. Ultrasonido y cáncer de ovario. Caracterización subjetiva. Rev Chil Obstet Ginecol 2005: 70 (5): 328-331.

14. Amor F., Vaccaro H. GIRADS o una propuesta de clasificación e informe de las imágenes anexiales por ultrasonografía transvaginal. Revista Chilena de Ultrasonografía 2007; 10(2): 75-76. 